\title{
Similarity of Traveling-Wave Delays in the Hearing Organs of Humans and Other Tetrapods
}

\author{
Mario A. Ruggero and Andrei N. Temchin \\ Department of Communication Sciences and Disorders, The Hugh Knowles Center \& Institute for Neuroscience, Northwestern \\ University, 2240 Campus Drive, Evanston, IL 60208, USA
}

Received: 4 November 2006; Accepted: 16 March 2007; Online publication: 31 March 2007

\begin{abstract}
Transduction of sound in mammalian ears is mediated by basilar-membrane waves exhibiting delays that increase systematically with distance from the cochlear base. Most contemporary accounts of such "traveling-wave" delays in humans have ignored postmortem basilar-membrane measurements in favor of indirect in vivo estimates derived from brainstem-evoked responses, compound action potentials, and otoacoustic emissions. Here, we show that those indirect delay estimates are either flawed or inadequately calibrated. In particular, we argue against assertions based on indirect estimates that basilar-membrane delays are much longer in humans than in experimental animals. We also estimate in vivo basilar-membrane delays in humans by correcting postmortem measurements in humans according to the effects of death on basilar-membrane vibrations in other mammalian species. The estimated in vivo basilar-membrane delays in humans are similar to delays in the hearing organs of other tetrapods, including those in which basilar membranes do not sustain traveling waves or that lack basilar membranes altogether.
\end{abstract}

Keywords: cochlea, basilar membrane, auditory, otoacoustic emissions, compound action potentials, brainstem evoked responses

Correspondence to: Mario A. Ruggero • Department of Communication Sciences and Disorders, The Hugh Knowles Center \& Institute for Neuroscience • Northwestern University • 2240 Campus Drive, Evanston, IL 60208, USA. Telephone: +1-847-4913180; fax: +1-8474912523; email: mruggero@northwestern.edu

\section{INTRODUCTION}

Transduction of sound in mammalian ears is mediated by basilar-membrane (BM) waves exhibiting delays that increase systematically with distance from the cochlear base. Fragmentary estimates of such traveling-wave delays exist for several species [see Table 2 in Robles and Ruggero (2001)]. Recently, a nearly complete map of delays, the first for any mammalian species, was drafted on the basis of correlated in vivo BM and auditory nerve measurements in chinchilla (Temchin et al. 2005). Siegel et al. also presented tentative maps for the cochleae of cats and guinea pigs (Siegel et al. 2005). A map of cochlear delays in living humans would be of immense interest but it is impossible to obtain using in vivo measurements such as recordings of BM vibrations or individual auditory nerve fibers: such recordings are highly invasive using current methods and will probably remain undoable in the foreseeable future.

Delays of BM waves were first measured in the temporal bones of human cadavers more than 60 years ago (von Békésy 1943), but, with rare exceptions, most contemporary accounts of BM delays in humans have ignored measurements in temporal bones in favor of indirect estimates based on brainstem-evoked responses (BSERs), compound action potentials (CAPs), and otoacoustic emissions (OAEs). Some of those estimates led to assertions that BM delays are much longer in humans than in common experimental animals (Neely et al. 1988; Shera et al. 2002). Here, we dispute those assertions, showing that all indirect estimates of BM delays in humans, including those used to justify the assertions, are flawed or are not yet adequately calibrated 
against $\mathrm{BM}$ measurements. We also show that estimates of in vivo BM delays in human cochleae, obtained by correcting postmortem BM data according to the effects of death on BM vibrations in experimental animals, are very similar to delays in the hearing organs of other mammals and in nonmammalian tetrapods, including those in which BMs do not sustain traveling waves or that lack BMs altogether.

\section{MATERIALS AND METHODS}

Estimates of cochlear delays in humans and experimental animals were drawn from the literature. Delay estimates not explicitly stated in texts or tables were computed from data extracted from figures by digitization (digiMatic, FEB Software, Chesterfield, VA, USA).

\section{RESULTS}

Measures of BM delays

In the analysis of linear systems, three measures of delay are commonly used (Fig. 1): phase, signal-front, and group [pp. 134-136 of Papoulis (1962)]. Although BM responses in healthy cochleae are nonlinear, the same concepts apply (Goldstein et al. 1971; Recio and Rhode 2000; Ruggero 1980). The phase delay is the phase lag at any given frequency expressed in units of time (Fig. 1C). The signal-front delay, a frequency-independent delay, is the latency of the impulse response (Fig. 1A). In many linear systems, the signal-front delay can be measured as equally well as the negative of the high-frequency asymptotic slope of the phase-vs.-frequency function (Fig. 1C) (Brillouin 1960; Goldstein et al. 1971; Papoulis 1962; Recio et al. 1998; Ruggero 1980). However, this procedure cannot be performed using BM data because its phase curves are terminated by high-frequency plateaus (Robles and Ruggero 2001). Other authors have obtained estimates of signalfront delays from the slope of the low-frequency segment of phase-vs.-frequency curves (Robles et al. 1976; Ruggero 1994). Although such estimates correctly matched the directly measured signal-front delays at the $7-\mathrm{kHz}$ site of the squirrel monkey cochlea (Robles et al. 1976), theoretical considerations indicate that they overestimate the signal-front delays measured with clicks (Papoulis 1962); see also Figure 1 of (Ruggero 1980).

In experimental animals, the signal-front delay remains unaltered postmortem at both basal [e.g., Fig. 1 of Ruggero (1994); Fig. 14 of Recio et al. (1998)] and apical (Cooper and Rhode 1996; Zinn et al.
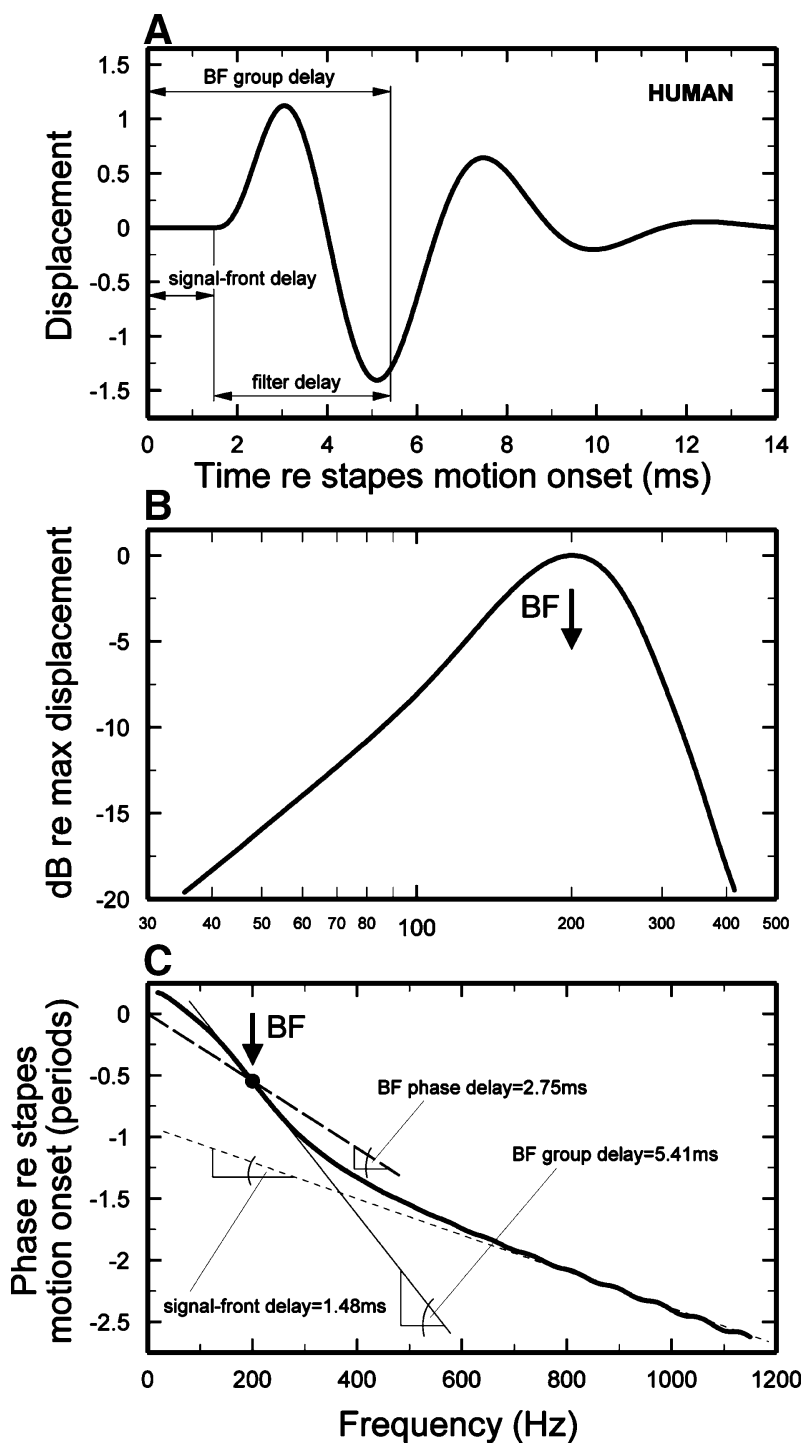

FIG. 1. Delays in BM vibrations at the $200-\mathrm{Hz}$ site of the human cochlea. A Impulse response synthesized on the basis of von Békésy's measurements of the latencies of BM responses to clicks (von Békésy 1949a) and of the magnitudes and phases of responses to tones (von Békésy 1947) in the cochleae of human cadavers. The signal-front delay is the latency of cochlear responses to clicks, measured from the onset of stapes motion [Fig. 11 of von Békésy (1949a)]. The BF group delay, the center of gravity of the impulse response, equals the signal-front delay plus the filter delay. The filter delay corresponds to a minimum-phase impulse response [Figs. 7 and 9 of Flanagan and Bird (1962)] computed on the basis of responses to tones (von Békésy 1947). B Spectral magnitudes of the impulse response of A. C Phase-vs.-frequency curve for the impulse response of $\mathbf{A}$. The negative slope around $\mathrm{BF}(\mathbf{C})$ is identical to the center of mass of the impulse response (A). The negative slope of the high-frequency asymptote $(\mathbf{C})$ is identical to the signal-front delay (A). Phase delay is the phase lag expressed in units of time or, equivalently, the negative slope of a straight line connecting a phase-frequency point and the origin (C).

2000) cochlear sites. In other words, the signal-front delay is a "passive" property of BM responses [see review by Ruggero (1994)]. Following von Békésy, we equate signal-front delay to BM travel time [see 
Fig. 11 of von Békésy (1949a) and p. 2293 of Recio and Rhode (2000)]. Hence, in vivo traveling-wave delay can be measured postmortem, as von Békésy did in the case of the human cochlea [Fig. 11 of von Békésy (1949a)].

The group delay at any particular frequency is the negative of the slope of the phase-vs.-frequency function at that frequency. For BM responses to low-level stimuli in mature healthy cochleae, the $\mathrm{CF}$ group delay coincides with the weighted-average group delay (the "center of mass") of the impulse response. [Following Overstreet et al. (2002a, b), we distinguish between best frequency $(\mathrm{BF})$ and characteristic frequency $(\mathrm{CF})$. Best frequency is the stimulus frequency producing the largest response (without regard to stimulus level or cochlear health or development). Characteristic frequency is the BF of responses to low-level stimuli in mature and healthy cochleae.] For higher stimulus levels at the base of the cochlea, both the CF group delay and the weightedaverage group delay become shorter, the latter at a greater rate than the former due to the increasing weight of low-frequency regions, which have low group delays [see Fig. 1 of Ruggero (1994)]. In BM responses, the CF group delay is partly an "active" property because its exact value in vivo depends on the health of the cochlea and on the stimulus level (Recio et al. 1998; Ruggero et al. 1997). Postmortem, the CF group delay does not vary as a function of stimulus level and is nearly the same as the CF group delay for very intense stimuli in vivo.

The phase delay, signal-front delay, and BF group delay are illustrated in Figure 1, which is based on von Békésy's BM measurements in human temporal bones at the cochlear site with $\mathrm{BF}$ of $200 \mathrm{~Hz}$. Figure $1 \mathrm{~A}$ shows a delayed version of a minimum-phase impulse response [Figs. 7 and 9 of Flanagan and Bird (1962) ] computed by Flanagan and Bird on the basis of von Békésy's amplitude data for responses to tones (von Békésy 1947). The minimum-phase impulse response has been delayed by $1.48 \mathrm{~ms}$, the travel time (i.e., the signal-front delay) measured by von Békésy using responses to clicks [Fig. 11 of von Békésy (1949a) ]. After the signal-front delay, the BM "rings" with a period of $5 \mathrm{~ms}(=1 / \mathrm{BF}$; Fig. 1B). Note that the near-BF group delay is the sum of the signalfront delay and a filter delay [or "resonance build-up time" (de Boer and Nuttall 1996)].

\section{BM delays in experimental animals}

Direct measurements of BM delays in healthy cochleae are available only for a few basal sites in several mammalian species [for review, see Robles and Ruggero (2001)] and single apical sites in chinchilla (Cooper and Rhode 1996) and guinea pig (Zinn et al.
2000). The direct measurements in chinchilla were recently supplemented by extensive estimates of BM delays derived from responses of auditory nerve fibers (Temchin et al. 2005). The signal-front and CF group delay estimates derived from auditory nerve fibers, fully consistent with direct BM measurements at both basal and apical sites, have permitted drafting nearly complete maps of cochlear delays in chinchilla, the first for any species (Temchin et al. 2005). The $\mathrm{CF}$ group delay maps are indicated by the black solid lines in Figures 2A, 7, and 8. A CF group delay map for cat (solid and long-dash lines in Fig. 2C and blue solid lines in Fig. 8), still uncorroborated by direct BM recordings, was proposed (Siegel et al. 2005) on the basis of published auditory nerve data (Carney and Yin 1988; van der Heijden and Joris 2003; van der Heijden and Joris 2005). A more conjectural map for guinea pig (solid line of Fig. 2B and green solid line in Fig. 8) was drafted (Siegel et al. 2005) by adjusting an equation with the same form as the equations for cat and chinchilla to fit direct BM measurements at several basal sites and a single apical site (Zinn et al. 2000).

The putative derivation of BM delays in humans from OAE group delays

Shera et al. (2002) used the group delays of stimulusfrequency OAEs (SFOAEs) to estimate the CF group delays of $\mathrm{BM}$ responses and concluded that $\mathrm{BM}$ delays are much longer in humans than in common experimental animals [e.g., three times longer than in cats or guinea pigs for the $3-\mathrm{kHz}$ cochlear site according to Fig. 2 of Shera et al. (2002)]. They based that conclusion on the fact that SFOAE group delays are much longer in humans than in experimental species (Shera et al. 2002) and on the assumption that "the group delay of SFOAEs is equal to twice the group delay of the BM mechanical transfer function evaluated at the cochlear location with CF equal to the stimulus frequency" [page 3319 of Shera et al. (2002)]. Recently, Siegel et al. (2005) tested the assumption of Shera et al. (2002) by comparing SFOAE and BM group delays in chinchilla, cat, and guinea pig and found that, in those species, SFOAEs actually have group delays either similar to or lower than the near-CF BM group delays. That finding contradicts the aforementioned assumption [page 3319 of Shera et al. (2002)] for the species tested and suggests that the assumption is also invalid for estimating BM delays in other species, including humans.

Some investigations estimated $\mathrm{BM}$ delays in humans using the group delays of $2 \mathrm{f}_{1}-\mathrm{f}_{2}$ distortionproduct OAEs (DPOAEs) stimulated by one tone with fixed frequency and another with variable frequency (Bowman et al. 1997; Ramotowski and 

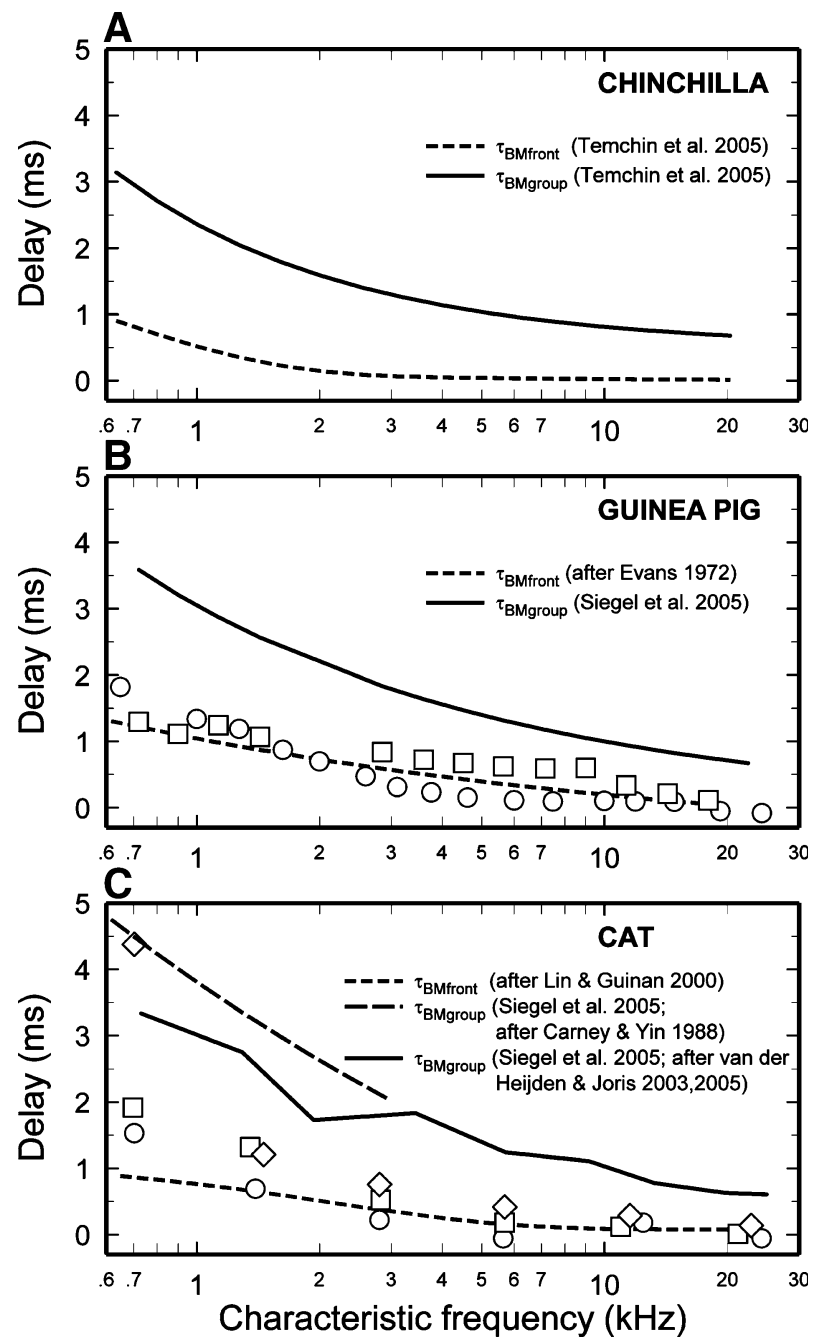

FIG. 2. Cochlear delays in chinchilla, cat, and guinea pig. Estimates of BM signal-front (short-dash lines) and CF group delays (solid and long-dash lines) based on direct BM recordings and/or recordings from auditory nerve fibers. [Note that the delays of responses of auditory nerve fibers corrected for neural/synaptic delays of $1 \mathrm{~ms}$ coincide with the delays of direct BM recordings (Temchin et al. 2005).] A Average signal-front and CF group delays for chinchilla BM (Temchin et al. 2005). B Derived-band estimates for guinea pigs from Figure 6 of Shore and Nuttall (1985) (click level is not specified) and Figure 3 of Aran and Cazals (1978) [click level is $65 \mathrm{~dB}$ pe SPL (50 dB HL)]. Solid line, from Figure 7A of Siegel et al. (2005). Dashed line, from data of Figure 1 of Evans (1972). C Derived-band delay estimates (open symbols) from a single cat from Figure 3 of Evans and Elberling (1982). Clicks were presented at 30,50, and $70 \mathrm{~dB}$ above the CAP threshold (defined as the level that evoked a CAP with average peak-to-peak amplitude of $5 \mu \mathrm{V}$ ). Solid and long-dash lines from Figure 7B of Siegel et al. (2005), based on data of Carney and Yin (1988), van der Heijden and Joris (2003), and van der Heijden and Joris (2005). Short-dash line, from Figure 6A of Lin and Guinan (2000).

Kimberley 1998; Schoonhoven et al. 2001). The estimates were based on the assumption that the DPOAE group delays for $f_{2}$ or $f_{1}$ sweeps amount to twice the $\mathrm{CF}$ group delay of the BM traveling wave. Such assumption has been shown to be invalid for chinchilla, guinea pig, and gerbil, in which DPOAE and BM group delays are actually similar (Gong et al. 2005; Narayan et al. 1998; Ren 2004; Ren et al. 2006; Ruggero 2004). Thus, it is likely that the assumption is also invalid for humans.

The putative derivation of BM delays in humans from BSER latencies

Neely et al. (1988) estimated BM delays in humans from the wave- $V$ latencies of BSERs to tone-bursts. Latencies were assumed to consist of sums of a constant (i.e., independent of stimulus level and frequency) neural component and a BM component, which varied strongly with stimulus level and frequency. Neely et al., recognizing that their BM delay estimates [e.g., $9 \mathrm{~ms}$ for $1-\mathrm{kHz}$ tones presented at $20 \mathrm{~dB}$ sound pressure level (SPL)] were "much longer than most previously reported values," suggested that humans have "longer cochlear travel times than most experimental animals" because "human subjects have longer, more compliant cochleas" (Neely et al. 1988). We show here that the long delay estimates actually resulted from flawed methodology and interpretation.

The interpretation of their data by Neely et al. (1998) was flawed because they incorrectly assumed that the large variation of BSER latency with stimulus level reflects solely BM nonlinearities and, therefore, that BM latencies can be simply estimated from changes in BSER latencies that accompany changes is stimulus levels. In fact, the variation of latency as a function of stimulus level is substantially smaller for $\mathrm{BM}$ or inner hair cell responses to $\mathrm{CF}$ tone bursts than the variations reported by Neely et al. (1988) for BSERs. For example, for $800-\mathrm{Hz}$ tones, BSER latencies decreased by $5.4 \mathrm{~ms}$ in the range $30-90 \mathrm{db}$ SPL [Fig. 2 of Neely et al. (1988)]. In the same level range, inner hair cell receptor potentials elicited by $800-\mathrm{Hz}$ (CF) tones change by only $2.2 \mathrm{~ms}$ [as estimated from Fig. 5 of Dallos (1985)], i.e., less than half of the latency change in BSERs. In the range $30-70 \mathrm{~dB}$ SPL, BSER latencies to $8-\mathrm{kHz}$ tones change by $1.6 \mathrm{~ms}$. In contrast, BM latencies in responses to $10-\mathrm{kHz} \mathrm{CF}$ tones [measured at a velocity of $0.1 \mathrm{~mm} / \mathrm{s}$ from Fig. 1 of Ruggero et al. (1997)] change by only $0.7 \mathrm{~ms}$, or less than half the latency change in BSERs for $8 \mathrm{kHz}$ tones. To summarize, the BSER intensity-dependent latency changes in humans amount to at least twice the corresponding $\mathrm{BM}$ or inner hair cell latency changes in experimental animals.

From the foregoing, it is clear that at least one half of the measured BSER latencies either was a methodological artifact (see below) and/or originated at sites of the auditory pathway more central than the inner hair cells. Recordings from auditory nerve 

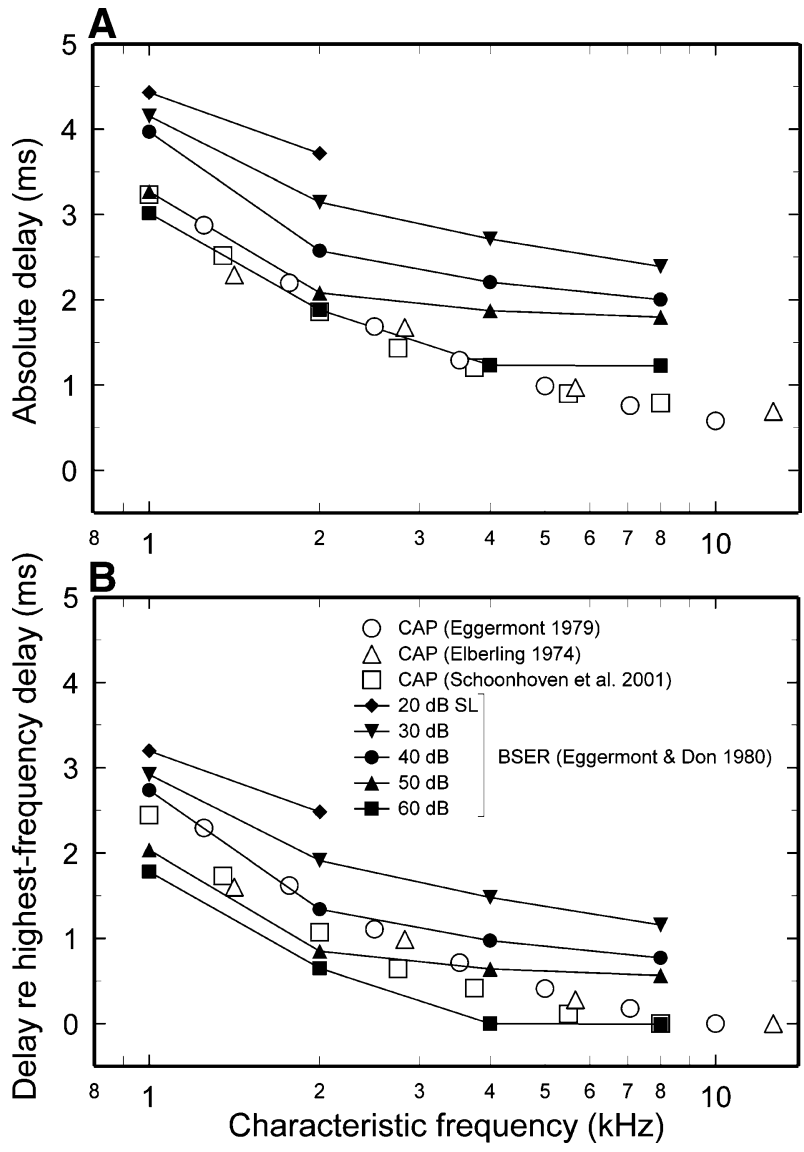

FIG. 3. Estimates of cochlear delays in humans based on derived-band CAPs and BSERs. A Estimates from CAPs: from Figure 4 of Elberling (1974), Figure 5 of Eggermont (1979), and Figure 3C of Schoonhoven et al. (2001). Estimates from BSERs: Figure 4 of Eggermont and Don (1980). Click levels were as follows: Elberling (1974), 95 dB p.e. SPL (i.e., "baseline-to-peak pressure corresponding to that of a tone with rms pressure of $95 \mathrm{~dB}$ SPL"); Eggermont (1979), $90 \mathrm{~dB}$ p.e. SPL; Schoonhoven et al. (2001), 98.4 p.e. SPL. The BM delay estimates of Eggermont (1979) and Schoonhoven et al. (2001) were calculated in the original publications on the assumption that synaptic/neural delays amount to $0.8 \mathrm{~ms}$. The delays estimated by Elberling (1974) were not corrected for synaptic/neural delays in the original publication and are presented here after subtraction of $0.8 \mathrm{~ms}$. B All conventions as for A, except that the derived-band measurements are presented relative to the minimum delay for each series of measurements.

fibers and cochlear nucleus neurons suggest that those sites are the synapses between inner hair cells and auditory nerve terminals. In the 30-90 dB SPL range, with onset stimulus ramps of $1.4 \mathrm{~ms}$, intensitydependent first-spike latency changes of auditory nerve fibers can be as large as $90 \mathrm{~ms}$ [Fig. 4A of Heil and Irvine (1997)]. In the same level range, using $6 \mathrm{~ms}$ on ramps, Kitzes et al. (1978) documented first-spike latency changes of about $30 \mathrm{~ms}$ in responses of cochlearnucleus neurons to $\mathrm{CF}$ tones $(11.6$ and $1.8 \mathrm{kHz})$ [Figs. 1 and 3 of Kitzes et al. (1978)].

The methodology of Neely et al. (1988) was flawed because the durations of the stimulus-onset ramps were not constant across stimulus frequencies. Brain- stem-evoked responses to tone bursts consist of neural activity synchronized by short stimulus-onset ramps. Therefore, to validly compare the latencies of responses to different tone bursts, the onset ramps must have a constant duration. In contrast, Neely et al. (1988) used tone bursts with onset-ramp durations that decreased as a function of increasing frequency (e.g., $4 \mathrm{~ms}$ for 0.25 and $0.5 \mathrm{kHz}, 2 \mathrm{~ms}$ for $1-2 \mathrm{kHz}, 1.4$ ms for $3 \mathrm{kHz}, 1 \mathrm{~ms}$ for $4 \mathrm{kHz}$, and $0.5 \mathrm{~ms}$ for $8 \mathrm{kHz}$ ), which should artificially produce delays that increase as stimulus frequency decreases (Neely et al. 1988). According to the aforementioned study by Heil and Irvine (1997) on auditory nerve fibers, for 30-dB SPL tones, lengthening ramp duration from 1.7 to $4.2 \mathrm{~ms}$ can increase first spike latency by $1.5-3$ ms [Figs. 2E and 3E of Heil and Irvine (1997)]. Between 0.5 and $3.5 \mathrm{kHz}$, Neely et al. (1988) found a BSER latency change of $5.9 \mathrm{~ms}$ for tone bursts presented at $30 \mathrm{~dB}$ SPL. The latency effects documented by Heil and Irvine suggest that between 1.5 and $3 \mathrm{~ms}$ (i.e., 25$50 \%$ ) of the 5.9-ms latency change can be attributed solely to differences in ramp duration (and, therefore, were not due to cochlear travel time). To summarize, the large dependencies of latency on stimulus frequency and level reported by Neely et al. include large, probably dominant, components that did not reflect BM latency shifts but rather were due to the different durations of the onset ramps and to level-dependent variations in first-spike latency of auditory nerve fibers.

\section{Estimation of cochlear delays in experimental animals using derived-band CAPS}

Teas et al. (1962) devised a so-called derived-band method that permits extracting some CF-specific information from click-evoked CAPs (Teas et al. 1962): CAPs evoked by clicks presented with a highpass noise are subtracted from CAPs evoked by clicks presented with a high-pass noise with higher-frequency cut-off. Such derived-band responses, presumably reflecting synchronized firings of auditory nerve fibers with CFs in the frequency range between the two cut-offs, have latencies that vary as a function of frequency in a manner reminiscent of BM delays (Teas et al. 1962).

Early comparisons of derived-band CAP latencies and responses to clicks of auditory nerve fibers in guinea pigs (Prijs and Eggermont 1981) and in a single cat (Evans and Elberling 1982) suggested that such latencies were CF-specific for CFs higher than 1$2 \mathrm{kHz}$ but could not test their accuracy rigorously because reliable measures of $\mathrm{BM}$ delays were not available. We now take advantage of newly available maps of BM delay for cat and guinea pig to compare them with derived-band delay estimates. 
Figure 2C permits comparing derived-band delay estimates for cat with BM CF group delays and signalfront delays, i.e., the latencies of auditory nerve fiber responses to intense rarefaction clicks, corrected for a neural/synaptic delay of $1 \mathrm{~ms}$ [see p. 389 of Ruggero and Rich (1987) and Figs. 2 and 7 of Siegel et al. (2005)]. For the cat cochlea, derived-band delays are shorter than CF BM group delays but similar to signal-front delays. For guinea pig (Fig. 2B), the derived-band delays also approximate the signal-front delays closely. These results, which are consistent with the strong skew toward short delays evident in responses to intense clicks at the BM (Recio et al. 1998) and (even more pronouncedly) in poststimulus histograms of auditory nerve fibers (Kiang et al. 1965; Lin and Guinan 2000), suggest that when implemented with intense stimuli the derived-band method estimates BM signal-front delays for CFs higher than 1-2 kHz.

\section{Derived-band delay estimates for the human cochlea}

Figure 3A shows BM delay estimates for humans based on derived-band CAPs measured with intense stimuli (open symbols) as well as BSERs measured with stimuli presented at several levels (filled symbols). The BM delay estimates were computed in the original publications by subtracting $0.8 \mathrm{~ms}$ from the latencies of the derived-band responses to clicks, on the assumption that synaptic and neural-conduction delays amount to $0.8 \mathrm{~ms}$. Observations listed below suggest that such a procedure is incorrect.

(1) In experimental animals, CAPs evoked by intense clicks principally reflect the activity of basal spiral ganglion cells. Therefore, the CAP latency matches the average first-spike latency of responses of high-CF auditory nerve fibers [e.g., Fig. 16 of Kiang (1984) and Fig. 5.14 of Kiang et al. (1965)]. (2) That latency, about $1 \mathrm{~ms}$, exceeds by about $1 \mathrm{~ms}$ the (very short) BM signal-front delay at the base of the cochlea [e.g., about $30 \mu$ s at the $10-\mathrm{kHz}$ place in chinchilla (Recio et al. 1998; Recio and Rhode 2000) ]. (3) As a corollary of 1 and 2, BM travel time (i.e., signal-front delay relative to stapes motion) to any given $\mathrm{CF}$ region can be accurately estimated by subtracting the latency of responses of high-CF fibers from the latency of responses of fibers with appropriate CF (Kiang et al. 1965; Ruggero and Rich 1987). (4) As another corollary of 1 and 2, BM travel time in experimental animals can be estimated equally well by subtracting the latency of derivedband CAPs for the highest CFs from derived-band estimates for lower CFs. (5) Presumably, the same procedure should apply equally well for estimating travel time from derived-band delays in humans.
Figure 3B shows the result of subtracting from the derived-band estimates of Figure $3 \mathrm{~A}$ the minimum latency in each of the corresponding experimental series, which generally corresponds to the derivedband latencies for the highest CF. For high CFs, the delays expressed relative to the delays at the most basal cochlear sites are, by definition, near zero. This result is consistent with the high traveling wave velocities at the base of the human cochlea (e.g., about $20 \mathrm{~m} / \mathrm{s}$ at the $10-\mathrm{kHz}$ place), as estimated from derivedband CAPs [see Fig. 9 of Eggermont (1976)]. For lower $\mathrm{CFs}$, the derived-band delays for humans grow larger.

The BSER derived-band estimates (Eggermont and Don 1980) of Figure 3B (filled symbols) appear to be the only ones that have addressed the intensity dependence of derived-band delays. For stimulation with intense clicks, derived-band delays measured with BSERs and CAPs are similar and very short (see also Schoonhoven et al. 2001). For lower click levels, delays grow systematically larger. The intensity dependence of derived-band BSER delays is qualitatively consistent with the large intensity dependence of the weighted-average group delay of responses to clicks of auditory nerve fibers [e.g., Figs. 5.9 and 5.10 of Kiang et al. (1965) ], which exaggerates the intensity dependence of BM weighted-average group delays [see Fig. 1 of Ruggero (1994)]. Thus, derived-band delays may reflect BM CF group delays at low stimulus levels and signal-front delays at intense levels. However, the precise relationship between BM delays and derivedband estimates cannot be specified at present with any certainty. Given such uncertainty, it seems clear that before derived-band CAPs can be used to estimate BM delays in humans, derived-band CAPs must first be adequately calibrated against BM delays in experimental animals, particularly with regard to their intensity dependence.

\section{BM delays in the cochleae of human cadavers}

Fortunately, it is not necessary to rely solely on indirect methods to estimate BM delays in the cochleae of living humans. Measurements of BM response phases in human cadavers have existed in the literature since 1943 (von Békésy 1943) but, with rare exceptions (Zwislocki 2002), contemporary discussions of cochlear delays in humans have either failed to take them into account (e.g., Bowman et al. 1997; Donaldson and Ruth 1993; Eggermont et al. 1991; Kimberley et al. 1993; Schoonhoven et al. 2001; Serbetcioglu and Parker 1999; Shera et al. 2002) or dismissed them as unrepresentative of in vivo mechanics [p. 652 of Neely et al. (1988)]. Phase-vs.frequency curves for postmortem BM responses in human cochleae at apical and at more basal sites are shown in Figures 1C and 4A, respectively. 
For comparison with the human data of Figure 4A, B shows phase-vs.-frequency curves for cochlear responses in squirrel monkey and chinchilla cochleae. The responses were recorded at sites with CFs comparable to those of Figure 4A. For squirrel monkey, phase-vs.-frequency curves are for postmortem BM responses at sites with BFs near $4.7 \mathrm{kHz}$ (solid lines and triangles) and in vivo responses (corrected for synaptic and conduction delays) of an auditory nerve fiber with $\mathrm{CF}$ of $2.3 \mathrm{kHz}$ to tones presented at 50 (open circles) and $90 \mathrm{~dB}$ SPL (dashed line). Figure $4 \mathrm{~B}$ also shows phase curves for chinchilla in vivo $\mathrm{BM}$
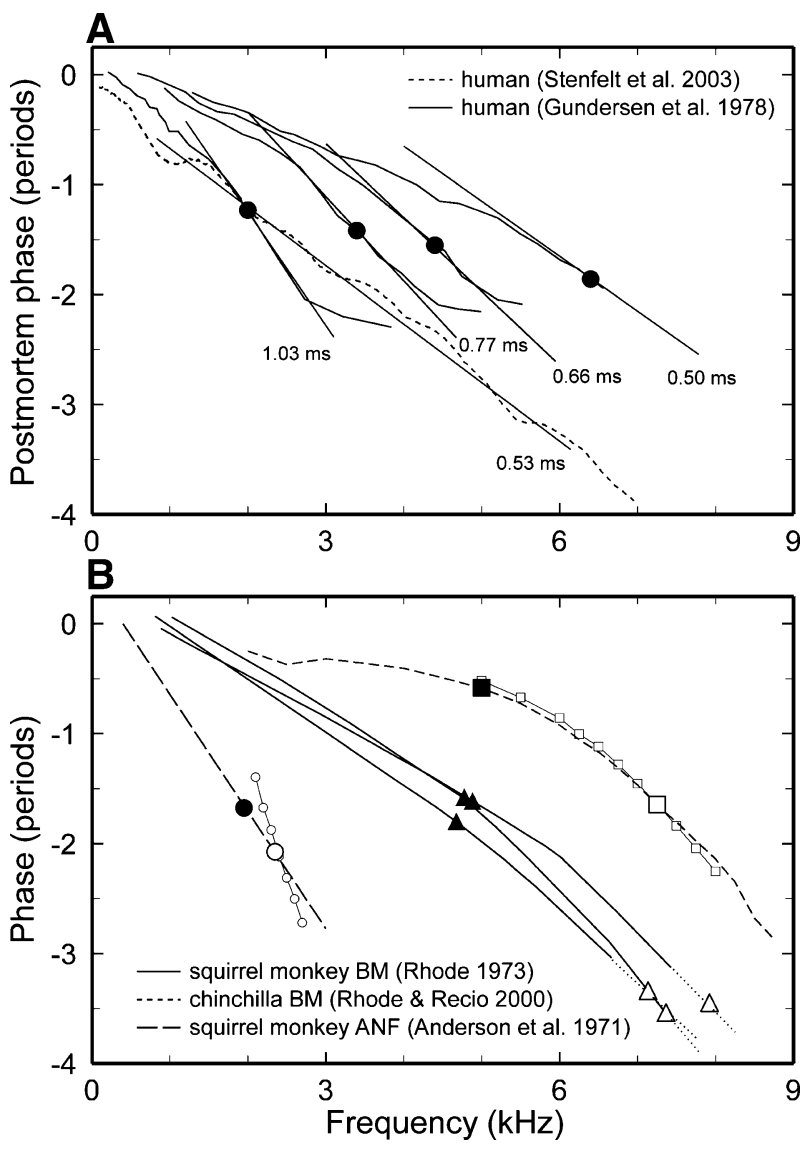

FIG. 4. Phase-vs.-frequency curves for cochlear responses in humans, squirrel monkey and chinchilla. A Phase-vs.-frequency curves for BM responses to tones in human cadavers; from Figure 3 of Gundersen et al. (1978) and Figure 5B of Stenfelt et al. (2003). Filled circles, BFs. The straight solid lines have slopes corresponding to BF group delays, indicated in milliseconds. B Phase-vs.-frequency curves for squirrel monkey and chinchilla cochleae. Data for squirrel monkey include postmortem BM responses at sites with BFs of about $4.7 \mathrm{kHz}$ [from Figs. 2, 3, and 4 of Rhode (1973)] and phase-vs.-frequency curves for responses of an auditory nerve fiber (ANF; CF: $2.3 \mathrm{kHz}$ ) to 50- (circles) and 90-dB SPL tones (long dashed line). To estimate BM phases, the ANF data [from Figs. 3 and 8 of Anderson et al. (1971)] were corrected for $1 \mathrm{~ms}$, the synaptic/neural conduction delay. The dotted lines are extrapolations of the measured curves. In vivo chinchilla BM responses to 30- (squares) and $80-\mathrm{dB}$ SPL (short-dash line) tones [from Figs. 2E and F of Rhode and Recio (2000)]. Large filled symbols, phase at BF. Large open symbols, phase at CF. responses to tones presented at 30 (open squares) and $80 \mathrm{~dB}$ SPL (short-dash line). The neural curves for squirrel monkey and the BM curves for chinchilla illustrate that, either in vivo or postmortem, CF group delays for responses to intense stimuli are shorter than in vivo responses to low-level stimuli. The responses for humans and squirrel monkey differ from those in chinchilla in having nearly straight-line phase-vs.-frequency curves. The difference between the curves for primates and rodents probably reflects both species dissimilarities and variation of the phase-vs.-frequency curves as a function of CF [see Pfeiffer and Molnar (1970) and p. 1319 of Robles and Ruggero (2001)].

The only direct measurements of signal-front delays for humans were obtained by George von Békésy in cadavers using intense click stimuli [Fig. 11 of von Békésy (1949a)]. The corresponding signalfront delays, shown in Fig. 5 (filled squares), are very similar to those in living chinchillas (dashed line) over the entire apical half of the cochlea and probably in the basal half as well, considering that both sets of data approach a 0-ms asymptote at high CFs. Figure 5 also shows the BF group delays of postmortem $\mathrm{BM}$ responses in the cochleae of humans (filled symbols) and experimental animals. Again, for any particular BF region, postmortem BF group delays are similar in all species, including humans.

\section{The effects of death on BM group delays}

To estimate in vivo CF group delays in humans from postmortem data, it is necessary to compensate for postmortem effects [reviewed in p. 1336 of Robles and Ruggero (2001)]. Figure 6A summarizes, for experimental animals, the effects on $\mathrm{BF}$ of death or, equivalently in vivo, of raising the stimulus level from low (i.e., the levels at which CF is measured) to high. Data points are included only when in vivo responses to low-level stimuli were measured in reasonably healthy cochleae. Specifically, Figure 6A shows in vivo $\mathrm{CFs}$ as a fraction of the postmortem BFs (or of the BFs of responses to intense stimuli). At the apex of the cochlea, postmortem BFs are nearly the same as (in vivo) CFs. At the base of the cochlea, CFs are $120-190 \%$ of postmortem BFs (mean $\sim 150 \%$ ). In other words, at the base of the cochlea, postmortem BFs are shifted downward by about 0.5 octave relative to in vivo CFs.

Figure $6 \mathrm{~B}$ summarizes the effects of death on the slopes of phase-vs.frequency curves for most of the measurements represented in Figure 6A. [Squirrel monkey data are not included because they are available only for cochleae exhibiting signs of deterioration.] Such effects consist of the abolition of the 


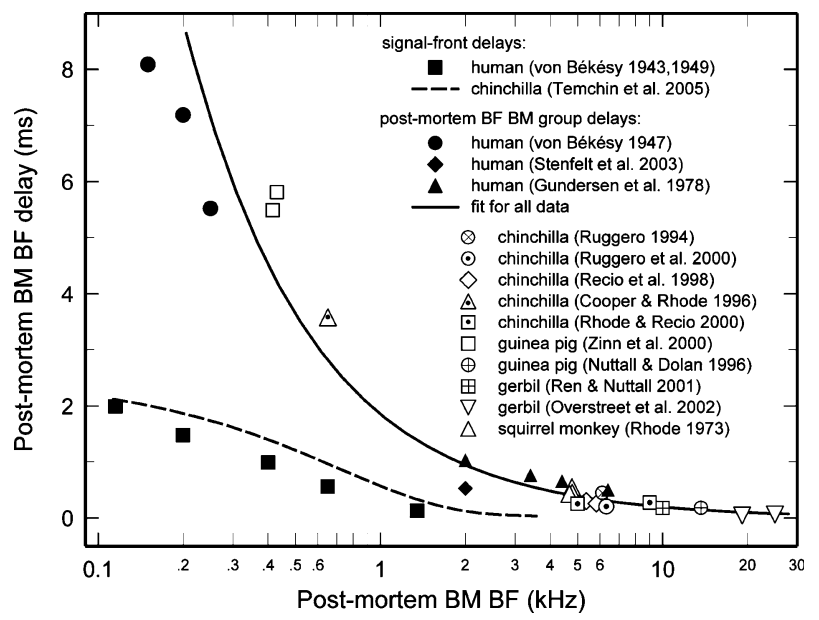

FIG. 5. Postmortem BM signal-front and BF group delays in the cochleae of humans and other mammals. Delays are expressed in milliseconds. Dashed lines, in vivo signal-front delays in chinchilla. Solid lines, the trend of postmortem BF group delays in all species. Signal-front delays in the cochleae of human cadavers from Figure 11 of von Békésy (1949a). Best frequency group delays in the cochleae of human cadavers: from Figures 4 and 5 of von Békésy (1947), Figure 5B of Stenfelt et al. (2003), and Figure 3 of Gundersen et al. (1978). Postmortem delays in chinchilla: Figure 1 of Ruggero et al. (2000), Figure 10 of Recio et al. (1998), Figure 7 of Cooper and Rhode (1996), Figures $1 \mathrm{~A}$ and 2D of Rhode and Recio (2000), and Figure 4 of Ruggero et al. (1992). Guinea pig: from Figure 6 of Zinn et al. (2000) and Figure 6 of Nuttall and Dolan (1996). Gerbil: from Figure 3 of Ren and Nuttall (2001) and Figures 4 and 5 of Overstreet et al. (2002a). Squirrel monkey: from Figures 2, 3, and 4 of Rhode (1973).

intensity dependence of group delay, which is longest for in vivo response to low-level stimuli and shortest for intense stimulation either in vivo or postmortem [Fig. 4B and pp. 1311-1312 of Robles and Ruggero (2001)]. For reference, Figure 6B includes a dashed line indicating equality between postmortem $\mathrm{CF}$ delay and in vivo CF delay for low-level stimulation; i.e., the line indicates a lack of dependence of $\mathrm{CF}$ phases on intensity, as would be the case for (hypothetical) linear responses. The effects of death (or, in vivo, of stimulus intensity) are seen in Figure $6 \mathrm{~B}$ as a divergence between the dashed line and the actual trend of in vivo $\mathrm{CF}$ delay as a function of postmortem CF delay (solid line).

Figure 7 shows the signal-front delays for living humans (open symbols) and chinchillas (thin dashed line), reproduced directly from Figure 5 (because they are not affected by death) and plotted at CFs corrected according to Figure 4A. The signal-front delays are similar in the two species. The trends of Figure $6 \mathrm{~A}$ and $\mathrm{B}$ for experimental animals were used to derive in vivo $\mathrm{CF}$ group delays in humans (filled symbols in Fig. 7) on the basis of postmortem BF group delays in humans (Fig. 4A). First, the postmortem $\mathrm{BFs}$ were translated into in vivo $\mathrm{CFs}$ according to the trend line of Figure 6A. Then, the postmortem
CF group delays (taken as identical to the postmortem CF group delays because of the near linearity of the phase-vs.-frequency curves for humans) were translated into in vivo CF group delays for low-level stimuli according to the trend line of Figure $6 \mathrm{~B}$. The derivation of in vivo CF group delays from postmortem BM group delay data is based on the assumption that the quantitative relationship between postmortem and in vivo CF delays is approximately the same across all mammalian species and therefore is also applicable to humans. (The "Discussion" section presents evidence that the applicability to human
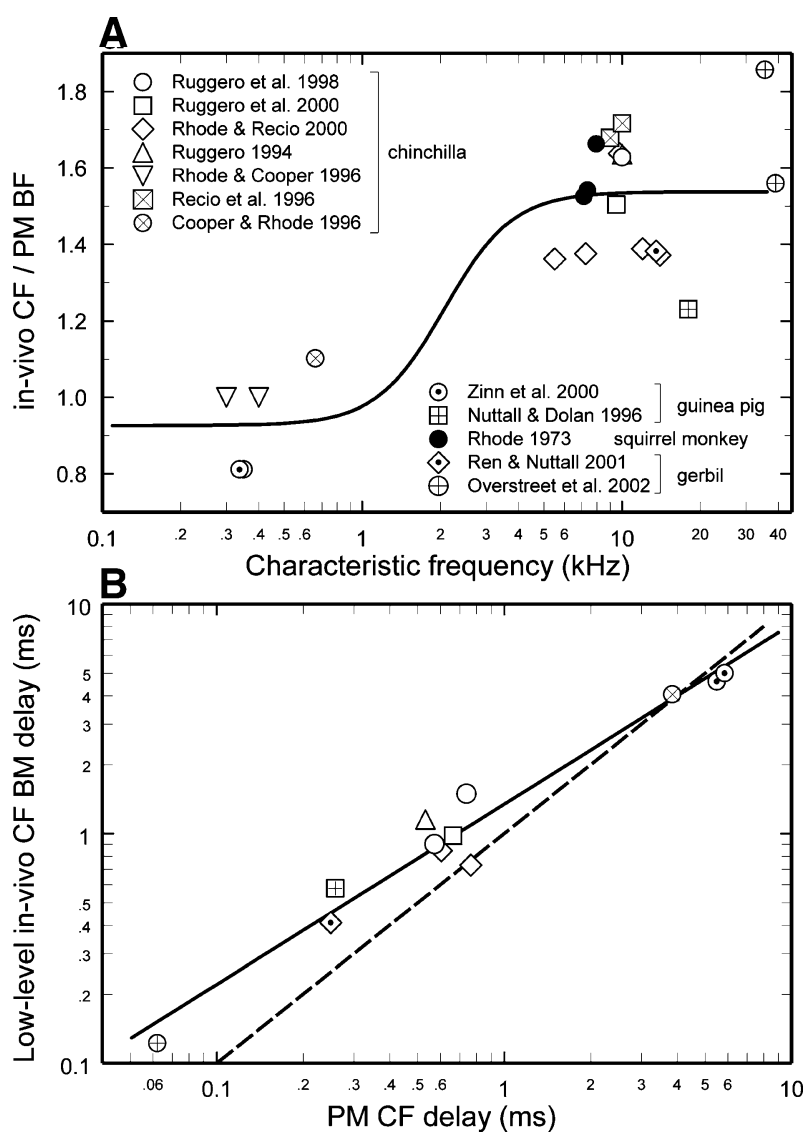

FIG. 6. In-vivo CFs and CF group delays for low-level stimuli in mammalian cochleae expressed as ratios of postmortem BFs and postmortem CF group delays. A In vivo CFs expressed relative to the BFs of responses to intense stimuli postmortem (plain symbols) or in vivo (dotted symbols). B The relationship between in vivo CF BM group delay for low-level stimuli and postmortem CF group delay in experimental animals. Solid line, trend between in vivo CF group delay for responses to low-level stimuli and postmortem CF group delay. Dashed line, equality between in vivo CF group delay for low-level stimulation and postmortem CF group delay. Chinchilla: from Figures 11A and B of Rhode and Cooper (1996), Figure 5 of Cooper and Rhode (1996), Figure 9 of Ruggero et al. (1997), Figure 1 of Ruggero et al. (2000), Figures 1C, 2B, 3B, and E of Rhode and Recio (2000), Figure 10 of Recio et al. (1998), and Figures 2 and 4 of Ruggero et al. (1992). Guinea pig: from Figure 6 and Table I of Zinn et al. (2000) and Figure 6 of Nuttall and Dolan (1996). Gerbil: from Figure 3 of Ren and Nuttall (2001) and Figures 4 and 5 of Overstreet et al. (2002a). 


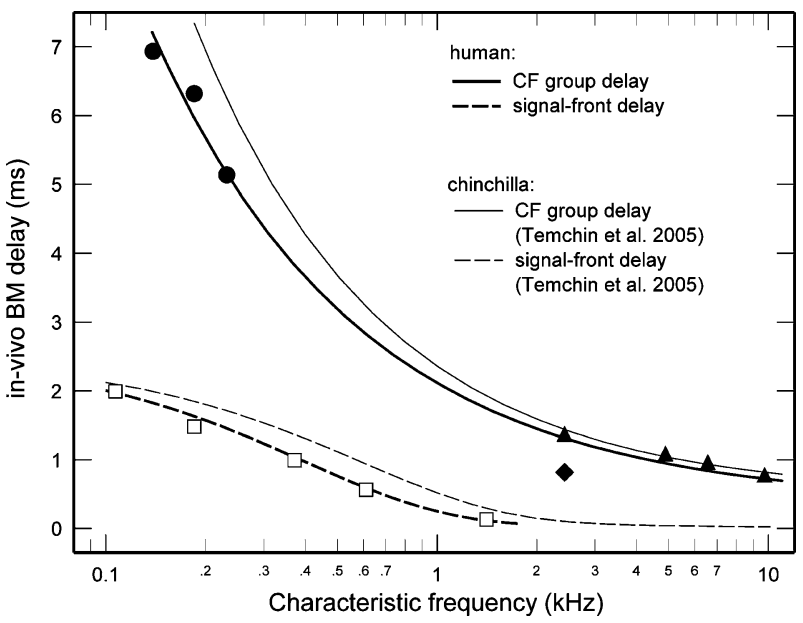

FIG. 7. In-vivo BM delays in the cochleae of humans and chinchillas. Open symbols indicate signal-front delays for humans, which are the same in vivo and postmortem. Chinchilla signal-front delays (thin dashed lines) from Figure 13A of Temchin et al. (2005). Filled symbols indicate in vivo CF group delays for humans obtained by correcting the postmortem data (Fig. 5) for the effects of death (Fig. 6). In vivo chinchilla near-CF group delays (thin solid line) from Figure 13B of Temchin et al. (2005).

cochleae of the quantitative relationship between postmortem and in vivo delays in the cochleae of experimental animals is not an arbitrary assumption but rather is founded on strong experimental evidence.)

Figure 7 also shows the trend (thin solid line) of in vivo $\mathrm{CF}$ group delays for low-level stimulation in chinchilla (Temchin et al. 2005). The in vivo CF group delays for humans and chinchillas are similar throughout most of the range of CFs. This similarity is of interest because the two species have comparable auditory thresholds and nearly identical hearing ranges [so that their cochlear maps are scaled versions of each other (Greenwood 1990)], but their BM lengths differ by a factor of nearly two (chinchillas, $18 \mathrm{~mm}$; humans, $35 \mathrm{~mm}$ ). The similarity of delays in spite of widely different BM lengths contradicts the conjecture that "the absolute magnitude of wave velocity in live human cochleae is roughly the same (as in others mammals)" [page 227 of Zwislocki (2002)] and its corollary, that because "human subjects have longer...cochleas" they must have "longer cochlear travel times than most experimental animals" [pp. 655-656 of Neely et al. (1988)]. [Caveat: Zwislocki's conjecture was based on an analysis of phase delay, a measure that can differ substantially from CF group delay (see Fig. 1).]

The minimal (or absent) dependence of BM delays on BM length is further illustrated in Figure 8, which shows that the signal-front (Fig. 8A) and CF group delays (Fig. 8B) of human cochleae are similar to the corresponding delays in the cochleae of other mammals (cat, guinea pig, and squirrel monkey, as well as chinchilla) with shorter BMs.
Similarity of CF group delays among mammalian and nonmammalian tetrapods

Signal-front and CF BM group delays in human cochleae (Fig. 8) are also similar to delays in the extremely short hearing organs of nonmammalian tetrapods [see Fig. 6B of Ruggero and Temchin (2005) ]. Such similarity is remarkable in the case of the bobtail lizard Tiliqua, whose BM does not support a traveling wave (Manley et al. 1988), and even more
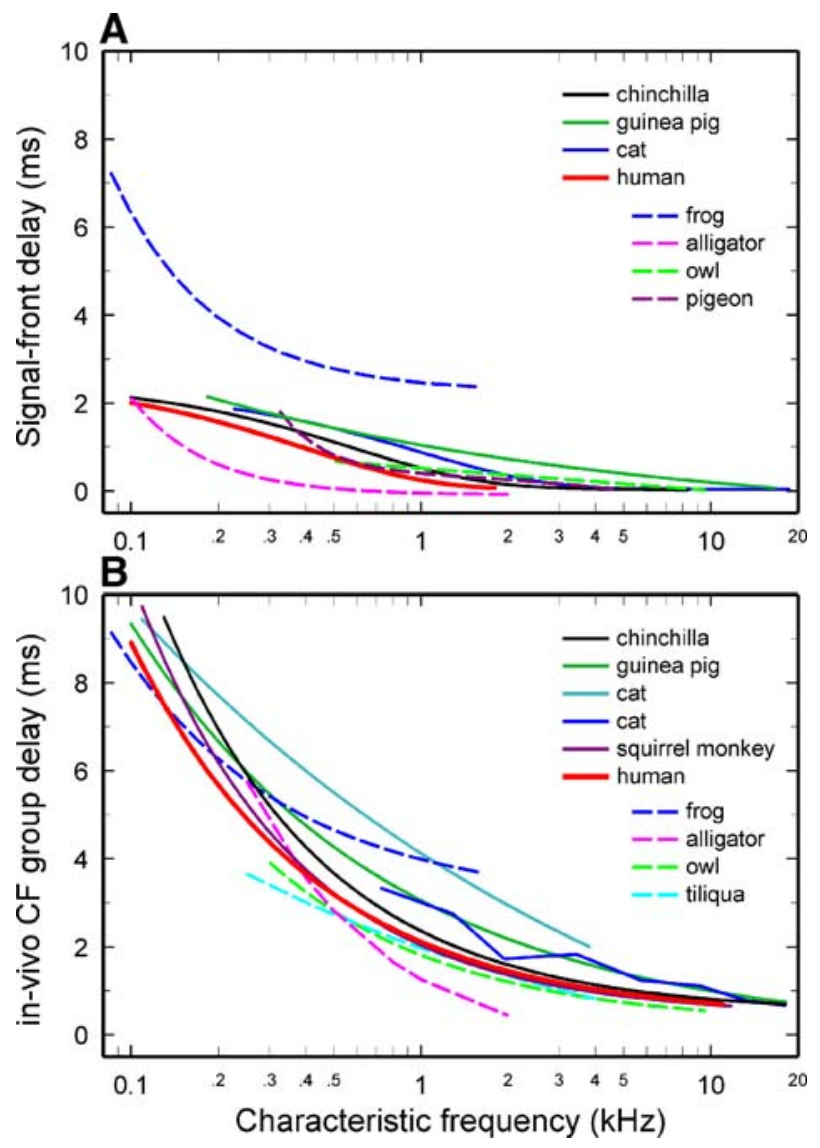

FIG. 8. Delays in the hearing organs of humans and other tetrapods. A Signal-front delays. B CF group delays. Chinchilla: Figure 13B of Temchin et al. (2005). Guinea pig: signal-front delay, Figure 1 of Evans (1972); group delay, Figure 7A of Siegel et al. (2005). Cat: Figure 7B of Siegel et al. (2005) after Carney and Yin (1988), van der Heijden and Joris (2003), and van der Heijden and Joris (2005). Frog: signal-front and group delay, Figure 14 of Hillery and Narins (1987). Alligator: group delay, Figure 11 of Smolders and Klinke (1986); signal-front delay, Figure 9 of Smolders and Klinke (1986). Tiliqua rugosa: data Figure 9A of Manley et al. (1990). Barn owl: group delay, Figure 10A (55-65 dB) of Köppl (1997b); signalfront delay, Figure 7 of Köppl (1997a). Pigeon signal-front delay: Figure 11 of Sachs et al. (1974). Monkey group delay: curve synthesized from responses of auditory nerve fibers with CFs lower than $1.5 \mathrm{kHz}$, from Figure 3 of Anderson et al. (1971), and BM responses at sites with CF $7-8 \mathrm{kHz}$, from Table I of Rhode (1978). In all cases, trend lines indicate CF-dependent cochlear mechanical delays (i.e., after correcting for middle-ear delays and, in the case of neural data, after correcting for synaptic and neural-conduction delays, as estimated in each publication). 
remarkable in the case of frogs (Hillery and Narins 1984; Hillery and Narins 1987), whose hearing organs lack BMs altogether. The implication is that systematic increases of signal-front and/or CF group delay as a function of decreasing $\mathrm{CF}$ do not necessarily reflect BM waves that travel from base to apex (Köppl 1997b; Manley 1990; Smolders and Klinke 1986).

\section{DISCUSSION}

Justification of the assumption that the effects of death on cochlear amplification and group delays in humans can be derived from the corresponding effects in experimental animals

The general assumption underlying the derivation of in vivo $\mathrm{CF}$ group delays from data in experimental animals is that cochlear processes in humans are similar to those in other mammalian species. Here we present evidence that the applicability to humans of quantitative relationships derived from experimental animals is not a mere assumption but rather a reasonable hypothesis supported by many facts. Specifically, we show below that (1) in vivo CFs and postmortem $\mathrm{BFs}$ are quantitatively related to each other in the same manner as they are in experimental species (Fig. 6A) and (2) in vivo CF group delays and postmortem CF group delays are quantitatively related to each other in the same manner as they are in experimental species (Fig. 6B).

Hypothesis 1 is supported by the phenomenon of "the half-octave shift": temporary threshold shifts induced by exposure to intense tones in humans (Davis et al. 1950) and other animals (Cody and Johnstone 1980) cause the greatest hearing-threshold elevations at frequencies one-half octave higher than that of the intense tone. It is almost certain that the half-octave shift reflects a corresponding shift in BM BF following death (Sellick et al. 1982) or stimulation with intense stimuli (Ruggero et al. 1996).

Hypothesis 2 is supported by the following facts.

(a) Postmortem, the peak gains of BM motion (i.e., at $\mathrm{BF}$ ) relative to incus or stapes motion are similar in humans and in experimental animals [humans, 10-25 dB, Figs. 2 of Gundersen et al. (1978) and $5 \mathrm{~A}$ of Stenfelt et al. (2003); squirrel monkey, 7-15 dB, Fig. 9 of Rhode (1978); gerbil, 26 dB, Fig. 6 of Overstreet et al. (2002a); and chinchilla, $20 \mathrm{~dB}$, Fig. 5 of Recio and Rhode (2000)].

(b) The peak magnitude of stapes or incus velocity gain (re stimulus pressure) is similar (in the order of $0.3 \mathrm{~mm} \mathrm{~s}^{-1} \mathrm{~Pa}^{-1}$ ) in humans and in experimental species [humans, Fig. 3 of Aibara et al. (2001); squirrel monkey, Figs. 3 and 4 of Rhode
(1971); gerbil, Fig. 2 of Overstreet and Ruggero (2002); chinchilla, Fig. 1 of Ruggero et al. (2007)].

(c) “...Most mammals, including humans, have the lowest (auditory) thresholds within $10 \mathrm{~dB}$ of $0 \mathrm{~dB}$ SPL" [pp. 7-8 of Fay (1994)]. In other words, with few exceptions, "the lowest level of stimuli that can be detected within the $\mathrm{BF}$ range is approximately the same for all mammalian species" [pp. 24-25 of Long (1994)].

(d) Postmortem changes in CF group delay are almost nil for low-CF sites (Cooper and Rhode 1996; Zinn et al. 2000).

(e) For high-CF sites, CF group delays are shortest postmortem or, in vivo, for intense stimuli; in vivo, $\mathrm{CF}$ group delays become systematically longer as stimulus levels are decreased or when cochlear health deteriorates [e.g., Fig. 2 of Ruggero (1994); reviewed on p. 1336 of Robles and Ruggero (2001)]. However, when $\mathrm{CF}$ group delay changes do occur postmortem, they are relatively small; e.g., $0.38 \mathrm{~ms}$ at the $10-\mathrm{kHz}$ site of chinchilla (Ruggero et al. 1997) or $0.25 \mathrm{~ms}$ at the $18 \mathrm{-kHz}$ site of guinea pig (Nuttall and Dolan 1996), corresponding to in vivo re postmortem gains at CF (i.e., "amplification") of 65 and $60 \mathrm{~dB}$, respectively. The ratio of postmortem group delay change to the corresponding gain change indicates the rate at which $\mathrm{CF}$ group delay varies with amplification: $5.8 \mu \mathrm{s} / \mathrm{dB}$ for chinchilla and $4.2 \mu \mathrm{s} / \mathrm{dB}$ for guinea pig.

From a, b, and c, it follows that the strength of cochlear amplification (the amount by which in vivo sensitivity exceeds postmortem sensitivity at CF) must be approximately the same in humans and other species. According to d, postmortem and in vivo $\mathrm{CF}$ group delay are the same for low $\mathrm{CF}$ regions in experimental animals and, hence, also probably in humans. According to e, even if large differences exist between humans and other species in the strength of cochlear amplification, those differences would be accompanied by relatively minor differences in CF group delay. For example, if amplification at $\mathrm{CF}$ were to amount in humans to $100 \mathrm{~dB}$ (i.e., $40 \mathrm{~dB}$ or two orders of magnitude higher than in chinchilla or guinea pig), the equation illustrated in Figure $6 \mathrm{~B}$ would underestimate by only $160-240 \mu \mathrm{s}$ (i.e., $40 \mathrm{~dB}$ times 4.2 or $5.8 \mu \mathrm{s} / \mathrm{dB}$ ) the $\mathrm{CF}$ group delays for CFs of $10-18 \mathrm{kHz}$.

Comparability of postmortem measurements of $\mathrm{BM}$ vibration in humans and in experimental animals

A matter of concern in extending observations in experimental animals to humans is whether the respective functional and structural properties are 
validly comparable. Postmortem observations in animal experiments (such as illustrated in Figs. 4-6) are typically carried out soon (within minutes or a few hours) after death, when cochlear structures may be presumed to remain largely intact. In contrast, BM vibration measurements in human temporal bones appear to have been carried out, at the earliest, several hours after death. Von Békésy stated that his vibration measurements were made in "fresh" temporal-bone preparations (von Békésy 1949b), but such explicit statements are lacking in von Békésy (1947) and von Békésy (1949a). The latter papers, however, refer to the use of "fresh" preparations in other contexts. Gundersen et al. (1978) gave no details regarding their temporal bone preparations, but another publication reporting on the same experiments states that measurements were carried in "temporal bones...prepared 8-24 h postmortem" (Kringlebotn et al. 1979). Stenfelt et al. (2003) made their BM measurements within 6 days after death in temporal bones that had been extracted 2 days after death and stored at $5^{\circ} \mathrm{C}$ in a $1: 10,000$ merthiolate solution in normal saline (Stenfelt et al. 2003).

The concern about the dissimilarity of cochlear conditions in human temporal bones and in experimental animals is somewhat allayed by the fact that most postmortem changes in BM responses in experimental animals, in particular the disappearance of nonlinear features (including the dependence of CF group delay on stimulus intensity; Fig. 6B), occur soon (within minutes) after death (Recio et al. 1998; Rhode 1973). Nevertheless, lingering changes in "passive" features were noted by Kohllöffel (1972) and Rhode (1973). Of special interest is that, after the initial postmortem effects (Fig. 5B), further changes (decreases) in near-CF group delays remained small for as long as $31 \mathrm{~h}$ in squirrel monkey [see Fig. 4 of Rhode (1973)]. Kohllöffel compared responses in guinea pig cochleae 4 and 7 days after death and found that there was "practically no effect of aging on the phase patterns" (Kohllöffel 1972).

Significance of the present findings for the concept of a traveling wave

In a 1954 paper, Wever, Lawrence, and von Békésy reconciled some of their views on the nature of the traveling wave. They stated that when the cochlea is stimulated with a tone, a BM "displacement wave seems to be moving up the cochlea. Actually...each element of the membrane is executing sinusoidal vibrations...different elements...executing these vibrations in different phases. This action can be referred to as that of a traveling wave, provided that...nothing is implied about the underlying causes. It is in this sense that Békésy used the term 'traveling wave'...” [pp. 511-
513 of Wever et al. (1954)]. More than half a century later, the nature of cochlear mechanical "traveling waves" remains a matter of argument, as demonstrated by a recent discussion in Portland, OR [see pp. 534539 of Nuttall et al. (2006)]. Nevertheless, we agree with many others that physical traveling waves propagate in the mammalian cochlea, carrying energy from base to apex. Such traveling waves apparently result from interactions between fluid inertia and the structural properties of the organ of Corti and the BM, principally the latter's elasticity. The evidence for the existence of traveling waves in mammalian cochleae is abundant [see reviews by Robles and Ruggero (2001) and Ruggero (1994)]. Numerous measurements of BM vibration [especially those of Russell and Nilsen (1997), Ren (2002), and Rhode and Recio (2000) ], of fluid pressure in scala tympani near the BM (Olson 1998) and of responses of auditory nerve fibers (Pfeiffer and Kim 1975) have been complemented and interpreted by substantial theoretical and/or modeling work on hydrodynamics (de Boer 1984; Lighthill 1981; Mammano and Nobili 1993; Peterson and Bogert 1950; Taber and Steele 1981; Zwislocki 1948).

The situation is less clear in nonmammalian hearing organs. In particular, substantial signal-front delays that increase from high-CF to low-CF regions (Fig. 8) exist in the hearing organs of the bobtail lizard (Tiliqua), in which the BM does not sustain a traveling wave (Manley 1990), and frogs, which lack BMs altogether. Obviously, the concept of the traveling wave cannot be applied to those hearing organs in the same sense that it applies to mammals (Robles and Ruggero 2001; Ruggero 1994). Nevertheless, mechanical traveling waves may well be sustained by structures other than BMs, such as the tectorial membrane in the amphibian papilla of frogs (Hillery and Narins 1984) and the tympanic membrane of locusts (Windmill et al. 2005).

\section{SUMMARY AND CONCLUSIONS}

Assertions based on SFOAEs and BSERs that BM delays are much longer in humans than in experimental animals, as well as estimates of BM delays based on DPOAEs, are probably invalid because they depend on assumptions that are invalid for experimental animals. The validity of derived-band estimates of BM delays in humans is uncertain. Derived-band delay estimates should be calibrated against BM delays in experimental animals. Signalfront and BF BM group delays in the cochleae of human cadavers are similar to the corresponding postmortem delays in other mammals (Fig. 5). Measurements in experimental animals of the effects of death or, in vivo, of the intensity-dependence of 
$\mathrm{BM}$ responses (Fig. 6), permit the estimation of $\mathrm{CF}$ group delays for living humans (Fig. 7) on the basis of BM delays in cadavers (on the assumption that the relationship between postmortem and in vivo delays is similar across species). Signal-front and CF group delays in the cochleae of living humans appear to be similar to the corresponding delays in chinchillas, other mammals, and even nonmammalian species, including those in which a BM traveling wave does not exist (Fig. 8).

\section{ACKNOWLEDGEMENTS}

Hongxue Cai, Mandy Cerka, Yun-Hui Fan, Luis Robles, Jon Siegel, and Joe Zwislocki provided helpful comments on previous drafts of this article. The authors were supported by NIH Grant DC-00419 from the National Institute on Deafness and Other Communication Disorders.

\section{REFERENCES}

Aibara R, Welsh JT, Puria S, Goode RL. Human middle-ear sound transfer function and cochlear input impedance. Hear. Res. 152:100-109, 2001.

Anderson DJ, Rose JE, Hind JE, Brugge JF. Temporal position of discharges in single auditory nerve fibers within the cycle of a sine-wave stimulus: frequency and intensity effects. J. Acoust. Soc. Am. 49:1131-1139, 1971.

Aran JM, Cazals Y. Electrocochleography: animal studies. In: Naunton RF and Fernández C (eds) Evoked Electrical Activity in the Auditory Nervous System. New York, Academic Press, pp. 239-257, 1978.

Bowman DM, Brown DK, Eggermont JJ, Kimberley BP. The effect of sound intensity on f1-sweep and f2-sweep distortion product otoacoustic emissions phase delay estimates in human adults. J. Acoust. Soc. Am. 101:1550-1559, 1997.

BriLlouin L. Wave Propagation and Group Velocity. New York, Academic Press, 1960.

Carney LH, Yin TC. Temporal coding of resonances by lowfrequency auditory nerve fibers: single-fiber responses and a population model. J. Neurophysiol. 60:1653-1677, 1988.

Cody AR, Johnstone BM. Single auditory neuron response during acute acoustic trauma. Hear. Res. 3:3-16, 1980.

CoOper NP, Rhode WS. Fast travelling waves, slow travelling waves and their interactions in experimental studies of apical cochlear mechanics. Aud. Neurosci. 2:289-299, 1996.

DALlos P. Response characteristics of mammalian cochlear hair cells. J. Neurosci. 5:1591-1608, 1985.

Davis H, Morgan CT, Hawkins Je, Galambos R, Smith FW. Temporary deafness following exposure to loud tones and noise. Acta Otolaryngol. Suppl. LXXXVIII:4-57, 1950.

DE Boer E. Auditory physics. Physical principles in hearing theory. II. Phys. Rep. 105:141-226, 1984.

de Boer E, Nuttall AL. Cochlear travel time and minimum phase. Assoc. Res. Otolaryngol. Mid-Winter Meet. Abstr. 19:57, 1996.

Donaldson GS, Ruth RA. Derived band auditory brain-stem response estimates of traveling wave velocity in humans. I: Normal-hearing subjects. J. Acoust. Soc. Am. 93:940-951, 1993.

EgGERMONT JJ. Analysis of compound action potential responses to tone bursts in the human and guinea pig cochlea. J. Acoust. Soc. Am. 60:1132-1139, 1976.
EgGermont JJ. Narrow-band AP latencies in normal and recruiting human ears. J. Acoust. Soc. Am. 65:463-470, 1979.

Eggermont JJ, Don M. Analysis of the click-evoked brainstem potentials in humans using high-pass noise masking. II. Effect of click intensity. J. Acoust. Soc. Am. 68:1671-1675, 1980.

Eggermont JJ, Ponton CW, Coupland SG, Winkelaar R. Maturation of the traveling-wave delay in the human cochlea. J. Acoust. Soc. Am. 90:288-298, 1991.

Elberling C. Action potentials along the cochlear partition recorded from the ear canal in man. Scand. Audiol. 3:13-19, 1974.

Evans EF. The frequency response and other properties of single fibres in the guinea-pig cochlear nerve. J. Physiol. (Lond.) 226:263-287, 1972.

Evans EF, Elberling C. Location-specific components of the gross cochlear action potential: an assessment of the validity of the high-pass masking technique by cochlear nerve fibre recording in the cat. Audiology 21:204-227, 1982.

FAY RR. Comparative auditory research. In: Fay RR and Popper AN (eds) Comparative Hearing: Mammals. New York, SpringerVerlag, pp. 1-17, 1994.

Flanagan JL, Bird CM. Minimum phase responses for the basilar membrane. J. Acoust. Soc. Am. 34:114-118, 1962.

Goldstein JL, BAER T, KIANg NYS. A theoretical treatment of latency, group delay and tuning. Characteristics for auditory nerve responses to clicks and tones. In: Sachs MB (ed) The Physiology of the Auditory System. Baltimore, National Educational Consultants, pp. 133-141, 1971.

Gong Q, Temchin AN, Siegel JH, Ruggero MA. Similarity of group delays of basilar-membrane vibrations and distortion-product otoacoustic emissions in chinchilla. Assoc. Res. Otolaryngol. Mid-Winter Meet. Abstr. 28:113, 2005.

GREENWOOD DD. A cochlear frequency-position function for several species-29 years later. J. Acoust. Soc. Am. 87:2592-2605, 1990.

Gundersen T, Skarstein O, Sikkeland T. A study of the vibration of the basilar membrane in human temporal bone preparations by the use of the Mossbauer effect. Acta Otolaryngol. 86:225-232, 1978.

HeIl P, Irvine DR. First-spike timing of auditory-nerve fibers and comparison with auditory cortex. J. Neurophysiol. 78:2438-2454, 1997.

Hillery CM, Narins PM. Neurophysiological evidence for a traveling wave in the amphibian inner ear. Science 225:10371039, 1984.

Hillery CM, Narins PM. Frequency and time domain comparison of low-frequency auditory fiber responses in two anuran amphibians. Hear. Res. 25:233-248, 1987.

KIANG NYS. Peripheral neural processing of auditory information. In: Darian-Smith I (ed) The Nervous System. Volume III: Sensory Processes, Part 2. Bethesda, American Physiological Society, pp. 639-674, 1984.

Kiang NYS, Watanabe T, Thomas EC, Clark LF. Discharge Patterns of Single Fibers in the Cat's Auditory Nerve. Cambridge, M.I.T. Press, 1965.

Kimberley BP, BRown DK, Eggermont JJ. Measuring human cochlear traveling wave delay using distortion product emission phase responses. J. Acoust. Soc. Am. 94:1343-1350, 1993.

Kitzes LM, Gibson MM, Rose JE, Hind JE. Initial discharge latency and threshold considerations for some neurons in cochlear nuclear complex of the cat. J. Neurophysiol. 41:1165-1182, 1978.

Kohllöffel LUE. A study of basilar membrane vibrations. II. The vibratory amplitude and phase pattern along the basilar membrane (postmortem). Acustica 27:66-81, 1972.

Köppl C. Frequency tuning and spontaneous activity in the auditory nerve and cochlear nucleus magnocellularis of the barn owl Tyto alba. J. Neurophysiol. 77:364-377, 1997a.

Köppl C. Phase locking to high frequencies in the auditory nerve and cochlear nucleus magnocellularis of the barn owl, Tyto alba. J. Neurosci. 17:3312-3321, 1997b. 
Kringlebotn M, Gundersen T, Krokstad A, Skarstein O. Noise-induced hearing losses. Can they be explained by basilar membrane movement? Acta Otolaryngol. Suppl. 360:98-101, 1979.

Lighthill J. Energy flow in the cochlea. J. Fluid Mech. 106:149-213, 1981.

Lin T, Guinan JJ JR. Auditory-nerve-fiber responses to high-level clicks: interference patterns indicate that excitation is due to the combination of multiple drives. J. Acoust. Soc. Am. 107:2615-2630, 2000.

LONG GR. Psychoacoustics. In: Fay RR and Popper AN (eds) Comparative Hearing: Mammals. New York, Springer-Verlag, pp. 18-56, 1994.

Mammano F, NobiLi R. Biophysics of the cochlea: linear approximation. J. Acoust. Soc. Am. 93:3320-3332, 1993.

Manley GA. Peripheral Hearing Mechanisms in Reptiles and Birds. Berlin, Springer-Verlag, 1990.

Manley GA, Yates GK, Köppl C. Auditory peripheral tuning: evidence for a simple resonance phenomenon in the lizard Tiliqua. Hear. Res. 33:181-189, 1988.

Manley GA, Yates GK, Köppl C, Johnstone BM. Peripheral auditory processing in the bobtail lizard Tiliqua rugosa. IV. Phase locking of auditory-nerve fibers. J. Comp. Physiol. [A] 167:129-138, 1990.

Narayan SS, Recio A, Ruggero MA. Cubic distortion products at the basilar membrane and in the ear canal of chinchillas. Assoc. Res. Otolaryngol. Mid-Winter Meet. Abstr. 21:181, 1998.

NeEly ST, Norton SJ, Gorga MP, Jesteadt W. Latency of auditory brain-stem responses and otoacoustic emissions using toneburst stimuli. J. Acoust. Soc. Am. 83:652-656, 1988.

Nuttall AL, Dolan, DF. Steady-state sinusoidal velocity responses of the basilar membrane in guinea pig. J. Acoust. Soc. Am. 99:1556-1565, 1996.

Nuttall AL, Ren T, Gillespie PG, Grosh K, de Boer E. Auditory Mechanisms: Processes and Models. Singapore, World Scientific, 2006.

OLson ES. Observing middle and inner ear mechanics with novel intracochlear pressure sensors. J. Acoust. Soc. Am. 103:34453463, 1998.

Overstreet EH, Ruggero MA. Development of wide-band middle ear transmission in the Mongolian gerbil. J. Acoust. Soc. Am. 111:261-270, 2002.

Overstreet EH, Temchin AN, Ruggero MA. Basilar membrane vibrations near the round window of the gerbil cochlea. J. Assoc. Res. Otolaryngol. 3:351-361, 2002a.

Overstreet EH, Temchin AN, Ruggero MA. Passive basilar-membrane vibrations in gerbil neonates: mechanical bases of cochlear maturation. J. Physiol. (Lond.) 545:279-288, 2002b.

PApoulis A. The Fourier Integral and its Applications. New York, McGraw-Hill, 1962.

Peterson LC, Bogert BP. A dynamical theory of the cochlea J. Acoust. Soc. Am. 22:369-381, 1950.

PFEIFFER RR, KIM DO. Cochlear nerve fiber responses: distribution along the cochlear partition. J. Acoust. Soc. Am. 58:867-869, 1975.

PFeIFfer RR, Molnar CE. Cochlear nerve fiber discharge patterns: relationship to the cochlear microphonic. Science 167:1614$1616,1970$.

Prijs VF, Eggermont JJ. Narrow-band analysis of compound action potentials for several stimulus conditions in the guinea pig. Hear. Res. 4:23-41, 1981.

Ramotowski D, Kimberley B. Age and the human cochlear traveling wave delay. Ear Hear. 19:111-119, 1998.

RECIO A, RHODE WS. Basilar membrane responses to broadband stimuli. J. Acoust. Soc. Am. 108:2281-2298, 2000.

Recio A, Rich NC, Narayan SS, Ruggero MA. Basilar-membrane responses to clicks at the base of the chinchilla cochlea. J. Acoust. Soc. Am. 103:1972-1989, 1998.

REN T. Longitudinal pattern of basilar membrane vibration in the sensitive cochlea. Proc. Natl. Acad. Sci. U. S. A. 99:17101-17106, 2002.
ReN T. Reverse propagation of sound in the gerbil cochlea. Nat. Neurosci. 7:333-334, 2004.

ReN T, NutTALL AL. Basilar membrane vibration in the basal turn of the sensitive gerbil cochlea. Hear. Res. 151:48-60, 2001.

Ren T, He W, Scott M, Nuttall AL. Group delay of acoustic emissions in the ear. J. Neurophysiol. 96:2785-2791, 2006.

RHODE WS. Observations of the vibration of the basilar membrane in squirrel monkeys using the Mössbauer technique. J. Acoust. Soc. Am. 49:1218-1231, 1971.

RHODE WS. An investigation of postmortem cochlear mechanics. In Møller AR (ed) Basic Mechanisms in Hearing. New York, Academic, pp. 49-63, 1973.

RHode WS. Some observations on cochlear mechanics. J. Acoust. Soc. Am. 64:158-176, 1978.

Rhode WS, Cooper NP. Nonlinear mechanics in the apical turn of the chinchilla cochlea in vivo. Aud. Neurosci. 3:101-121, 1996.

Rhode WS, Recio A. Study of mechanical motions in the basal region of the chinchilla cochlea. J. Acoust. Soc. Am. 107:33173332, 2000.

Robles L, Rhode WS, Geisler CD. Transient response of the basilar membrane measured in squirrel monkeys using the Mossbauer effect. J. Acoust. Soc. Am. 59:926-939, 1976.

Robles L, Ruggero MA. Mechanics of the mammalian cochlea. Physiol. Rev. 81:1305-1352, 2001.

RUGGero MA. Systematic errors in indirect estimates of basilar membrane travel times. J. Acoust. Soc. Am. 67:707-710, 1980.

RugGero MA. Cochlear delays and traveling waves: comments on 'Experimental look at cochlear mechanics'. Audiology 33:131142, 1994.

Ruggero MA. Comparison of group delays of 2f1-f2 distortion product otoacoustic emissions and cochlear travel times. Acoust. Res. Lett. Online 5:143-147, 2004.

Ruggero, MA, Narayan, SS, Temchin, AN, Recio, A. Mechanical bases of frequency tuning and neural excitation at the base of the cochlea: comparison of basilar-membrane vibrations and auditory-nerve-fiber responses in chinchilla. Proc. Natl. Acad. Sci. U. S. A. 97:11744-11750, 2000.

Ruggero MA, Rich NC. Timing of spike initiation in cochlear afferents: dependence on site of innervation. J. Neurophysiol. 58:379-403, 1987.

Ruggero MA, Rich NC, Recio A. Basilar membrane responses to clicks. In: Cazals Y, Demany L, and Horner K (eds) Auditory Physiology and Perception. London, Pergamon Press, pp. 8591, 1992.

Ruggero MA, Rich NC, Recio A. The effect of intense acoustic stimulation on basilar-membrane vibrations. Aud. Neurosci. 2:329-345, 1996.

Ruggero MA, Rich NC, Recio A, Narayan SS, Robles L. Basilarmembrane responses to tones at the base of the chinchilla cochlea. J. Acoust. Soc. Am. 101:2151-2163, 1997.

Ruggero MA, Temchin AN. Unexceptional sharpness of frequency tuning in the human cochlea. Proc. Natl. Acad. Sci. U. S. A. 102:18614-18619, 2005.

Ruggero MA, Temchin AN, Fan Y-H, Cai H. Boost of transmission at the pedicle of the incus in the chinchilla middle ear. In: Eiber A and Huber A (eds) Middle Ear Mechanics in Research and Otology. Singapore, World Scientific, pp. 160-163, 2007.

Russell IJ, Nilsen KE. The location of the cochlear amplifier: spatial representation of a single tone on the guinea pig basilar membrane. Proc. Natl. Acad. Sci. U. S. A. 94:26602664, 1997.

SAchs MB, Young ED, Lewis RH. Discharge patterns of single fibers in the pigeon auditory nerve. Brain Res. 70:431-447, 1974.

Schoonhoven R, Prijs VF, Schneider S. DPOAE group delays versus electrophysiological measures of cochlear delay in normal human ears. J. Acoust. Soc. Am. 109:1503-1512, 2001. 
Sellick PM, Patuzzi R, Johnstone BM. Measurement of basilar membrane motion in the guinea pig using the Mössbauer technique. J. Acoust. Soc. Am. 72:131-141, 1982.

Serbetcioglu MB, Parker DJ. Measures of cochlear travelling wave delay in humans: I. Comparison of three techniques in subjects with normal hearing. Acta Otolaryngol. 119:537-543, 1999.

Shera CA, Guinan JJ, Oxenham AJ. Revised estimates of human cochlear tuning from otoacoustic and behavioral measurements. Proc. Natl. Acad. Sci. U. S. A. 99:3318-3323, 2002.

Shore SE, NutTall AL. High-synchrony cochlear compound action potentials evoked by rising frequency-swept tone bursts. J. Acoust. Soc. Am. 78:1286-1295, 1985.

Siegel JH, Cerka AJ, Recio-Spinoso A, Temchin AN, Van Dijk P, Ruggero MA. Delays of stimulus-frequency otoacoustic emissions and cochlear vibrations contradict the theory of coherent reflection filtering. J. Acoust. Soc. Am. 118:2434-2443, 2005.

SMOLDERS JW, KLINKE R. Synchronized responses of primary auditory fibre-populations in Caiman crocodilus (L.) to single tones and clicks. Hear. Res. 24:89-103, 1986.

Stenfelt S, Puria S, Hato N, Goode RL. Basilar membrane and osseous spiral lamina motion in human cadavers with air and bone conduction stimuli. Hear. Res. 181:131-143, 2003.

TABer LA, Stekle CR. Cochlear model including three-dimensional fluid and four modes of partition flexibility. J. Acoust. Soc. Am. 70:426-436, 1981.

Teas DC, Eldredge DH, Davis H. Cochlear responses to acoustic transients: an interpretation of whole-nerve action potentials. J. Acoust. Soc. Am. 34:1438-1459, 1962.

Temchin AN, Recio-Spinoso A, van Dijk P, Ruggero MA. Wiener kernels of chinchilla auditory-nerve fibers: verification using responses to tones, clicks and frozen noise and comparison to basilarmembrane vibrations. J. Neurophysiol. 93:3635-3648, 2005.

VAN DER HeIJDen M, Joris PX. Cochlear phase and amplitude retrieved from the auditory nerve at arbitrary frequencies. J. Neurosci. 23:9194-9198, 2003.

VAN der HeIJden M, Joris PX. The speed of auditory low-side suppression. J. Neurophysiol. 93:201-209, 2005.

von BÉKÉsy G. Uber die Rezonanzkurve und die Abklingzeit der verschiedenen Stellen der Schneckentrennwand. Akust. Zeits. 8:66-76, 1943.

vON BÉKÉsy G. The variation of phase along the basilar membrane with sinusoidal vibration. J. Acoust. Soc. Am. 19:452-460, 1947.

VON BÉKÉSY G. On the resonance curve and the decay period at various points on the cochlear partition. J. Acoust. Soc. Am. 21:245-254, 1949a.

vON BÉKÉsY G. The vibration of the cochlear partition in anatomical preparations and in models of the inner ear. J. Acoust. Soc. Am. 21:233-245, 1949b.

Wever EG, Lahrence M, von BéKÉsy G. A note on recent developments in auditory theory. Proc. Natl. Acad. Sci. U. S. A. 40:508-512, 1954.

Windmill JFC, Gopfert MC, Robert D. Tympanal travelling waves in migratory locusts. J. Exp. Biol. 208:157-168, 2005.

Zinn C, Maier H, Zenner H, Gummer AW. Evidence for active, nonlinear, negative feedback in the vibration response of the apical region of the in-vivo guinea-pig cochlea. Hear. Res. 142:159-183, 2000.

ZwISLOCKI JJ. Theorie der Scheneckenmechanik: qualitative und quantitative analyse. Acta Otolaryngol. Suppl. 72:1-76, 1948.

ZwisLocki JJ. Auditory Sound Transmission-An Autobiographical Perspective. Mahwah, Lawrence Erlbaum Associates, 2002. 